\title{
Research Progress of Traditional Chinese Medicine Maintenance Treatment for Ulcerative Colitis during Remission
}

\author{
Xiya Zhu*, Li Liu, Xiaoquan Du \\ Affiliated Hospital of Shaanxi University of Traditional Chinese Medicine, Xianyang 712000, Shaanxi, China.
}

How to cite this paper: Xiya Zhu, Li Liu,
Xiaoquan Du. (2020) Research Progress of
Traditional Chinese Medicine Maintenance
Treatment for Ulcerative Colitis During
Remission. International Journal of
Clinical and Experimental Medicine
Research, 4(4), 254-259.
DOI: $10.26855 /$ ijcemr.2020.10.022

Received: October 18, 2020

Accepted: November 9, 2020

Published: November 17, 2020

*Corresponding author: Xiya Zhu, Affiliated Hospital of Shaanxi University of Traditional Chinese Medicine, Xianyang 712000, Shaanxi, China.

\begin{abstract}
Maintenance remission therapy has an important role in reducing the recurrence rate of ulcerative colitis, and the selection of appropriate drugs for maintenance therapy has attracted more and more clinical attention. Western medicine maintenance treatment has a long medication cycle, which is easy to produce changes in tolerance and drug resistance, and frequent adverse reactions bring unnecessary economic burden. With the continuous development of Chinese medicine, its efficacy in maintaining remission is no less than that of Western medicine, and it has the advantages of reducing recurrence, reducing adverse reactions, diverse treatment methods, and low treatment costs. This article reviews the current status of clinical research on maintenance treatment of ulcerative colitis in remission period.
\end{abstract}

\section{Keywords}

Remission period of ulcerative colitis, traditional Chinese medicine therapy, maintenance treatment, review

Ulcerative colitis (UC) is an intestinal inflammatory disease that does not heal for life and the causative factors are not yet fully clear. The clinical features are recurrent or persistent episodes of abdominal pain, diarrhea, mucus, pus and blood in the stool. Period and remission period alternate. It is currently believed that UC is based on genetic factors and can activate the immune and non-immune systems through infection, psychological stress and environmental factors, which in turn triggers changes in the structure of the intestinal mucosa and internal environment, and ultimately leads to chronic non-specific inflammation of the intestine [1, 2]. And research shows [3, 4], as the incidence UC western countries tends to stabilize, the prevalence rate of non-western countries represented by East Asia increases significantly.

\section{UC recurrence is an urgent problem in Chinese and Western medical circles}

At present, the clinical treatment rate of UC in China is high, but the remission rate is relatively low and the recurrence rate is high, which is worthy of attention and urgent to solve. Active period has attracted clinical attention because of its obvious clinical manifestations and parenteral complications, significant colonoscopy changes and pathological biopsy indicators. Although it has reached the maximum remission state in all aspects after various 
treatment induction, it cannot be used as the end point of UC patients' treatment. Although the remission period does not reach the state of "deep remission", it is still in the stage of latent disease, low body defense and easy to be induced by external causes. Although the condition tends to be stable, there is still the same pathological mechanismas the active period [5]. The pathological factors lurking in the intestines gradually reach quantitative changes to qualitative changes, which are the basis and "root" of UC recurrence. Therefore, attention to maintenance treatment in remission is an important way to solve recurrence [6]. While the maintenance of western medicine in patients with UC remission has gradually improved, there are still many problems such as: 5-ASA's poor curative effect during maintenance treatment, high recurrence rate, easy recurrence of inflammation after withdrawal, and persistent intestinal mucosal damage and repair and regeneration. Immunosuppressive therapy has not been accepted by most doctors and patients; economic factors restrict the application of biological agents; the application of probiotics and other drugs still needs evidence-based medicine, so it has not been widely [7]. Although the long-term effect UC traditional Chinese medicine treatment is not satisfactory, the remission period after withdrawal is relatively longer than that of western medicine, and the side effects of drugs are less. In recent years, some progress has been made in its maintenance and treatment. The comprehensive curative effect is improved and the clinical recurrence rate is obviously reduced, and the patient's body.

\section{Understanding of UC Retention Period in Modern Chinese Medicine}

Modern Chinese medicine scholars will take the UC, of attack and remission as the category of "rest and recuperation" of traditional Chinese medicine. In UC remission period, many pathological factors exist, resulting in repeated attacks. Clinically, spleen deficiency is the basis, dampness evil is the standard, its basic pathogenesis is to meet the deficiency, or deficiency in the solid, the most common in the deficiency [8].

\subsection{Spleen deficiency is the root}

There is an ancient record: "The root of diarrhea is due to the spleen and stomach... and dysentery is caused". The body with deficiency of spleen and gas, due to factors such as external feelings, diet, emotions, fatigue, etc., causes the spleen and stomach to lose function, and the internal dampness raw. Stagnation of dampness transforms heat, inhibits qi and blood, blood phase strokes, fat and collateral damage, blood sepsis and flesh rot into ulcers, and dysentery due to mixed red and white.

Modern immunology believes that the "spleen" in traditional Chinese medicine has a wide range of immunological connotations in terms of its physiological characteristics and functions. Anatomical and substantial spleen is the largest lymphatic reticuloendothelial system in human body and is closely related to human immune mechanism. "Spleen is the basis of acquired". It can nourish people's qi and improve their disease resistance. Associated with acquired immunity, that is, "spleen strong viscera difficult to hurt" [9, 10]. Clinically [11], many scholars have discussed the essential of "spleen deficiency syndrome" from the immunological mechanism that the development of immune organs such as spleen and thymus in animal model of spleen deficiency has been restricted to some extent. The damaged immune organ structure can be gradually restored to normal level by using Jianpi Yiqi prescription. Other studies have shown that [12] percentage of peripheral blood lymphocytes $\mathrm{T}$ spleen deficiency is significantly lower than the normal value, and the ratio of cell subsets is abnormal. It can be seen that spleen deficiency is associated with low state of immune system. Immune system is low or destroyed can cause intestinal inflammation, mucosal barrier damage stimulates intestinal immune system, cells and humoral immunity related to UC immunity are activated. Through a series of responses, cytokines with proinflammatory and mucosal damage were stimulated, and the release of other inflammatory factors was accelerated and synergistic when local tissues were induced to produce inflammation. One study showed that [13] low-grade inflammatory response of intestinal mucosa existed in some patients with UC remission, which was closely related to clinical recurrence.

\subsection{Wet evil is the second element}

On the basis of deficiency of physical endowment and damage of spleen and stomach, dampness evil is felt, or dampness is from endogenetic point of view. Wet for Yin evil, easy to attack Yang, but also easy to trap spleen, spleen Yang damage, the weaker qi, more susceptible to evil. Wet sticky, pathogenic lingering, repeated disease, difficult to heal. Spleen deficiency and dampness can be divided into order and influence each other throughout the course of disease. Intestinal microbes form intestinal mucosal barrier and regulate the expression of some genes and immune environment. When the biological barrier is broken, the flora becomes imbalanced, and the increase of pathogenic bacteria leads to intestinal epithelial cell damage, the integrity of the intestinal mucosa is destroyed, and the permeability increases, triggering inflammation and immune response. There are changes in the intestinal flora 
in the early stage of UC patients, which are the initiating and continuing factors of the disease [14]. Wen Xianghui et al. [15] explored the relationship between internal dampness and intestinal flora imbalance, and found that internal dampness can further lead to the imbalance of the flora. The same imbalanced microbiota can also participate in related immune inflammatory reactions, accelerating the growth of "internal dampness". In the pathological environment, the two are mutually causal. The method of clearing heat and removing dampness or transporting spleen in temperature can obviously improve and repair the unbalanced intestinal flora structure, thus effectively reverse the situation of internal dampness. Wang Jing [16] found that the colony values of Escherichia coli and bifidobacterium in the jejunum and colon of rats in the high and low humidity groups were different in the observation of artificial control of simulated environmental humidity, suggesting that external humidity can change the structure of the intestinal flora and affect Intestinal environment.

\subsection{Blood stasis is a pathological factor of recurrence}

Blood stasis is the main pathological factor in recurrent attacks during UC remission. Spleen disease persists for a long time to reach the kidneys, and the spleen and kidney yang deficiency and yin cold endogenous. The cold coagulation blood vessels and the qi nothingness promote the blood operation, resulting in the blood flow is not smooth and blood stasis, blood stasis exists, the new blood cannot be transformed, and finally the local formation of qi and blood deficiency to the blood deficiency of the vicious circle. Blood stasis is easy to knot with intestinal stagnation, brewing dampness and heat, increasing the recurrence rate of the disease. Studies have shown that [17] changes of blood flow dynamics in the intestinal mucosa of UC patients make the blood often in a hypercoagulable state, which leads to the long-term retention of local inflammatory factors located in the intestine. Thromboembolism in the intestine causes ischemia and necrosis of the intestinal mucosa, which leads to the enlargement of the scope of inflammatory lesions and the aggravation of ulcers, which accelerates the development of UC disease [18].

\section{Clinical Progress of Chinese Medicine UC Sustained Treatment}

\subsection{Self-made prescription oral treatment}

UC the relief period of patients with qi deficiency and evil qi has not been exhausted, lingering and easy to be induced, maintenance of remission treatment is also a long process. Oral Chinese medicine is effective in the treatment of the disease, and can be added and subtracted according to the change of the disease, and the medication cycle can also change flexibly with the course of disease, which ensures the long-term maintenance effect of different patients.

Zhang Tianbin and other [19] divided 56 patients with remission UC into treatment group 26 cases with oral decoction-free Jianpi Huoxuejiedu granule prescription (Codonopsis, Atractylodes macrocephala, Poria cocos, Fufangteng, Ulmus pumila, etc.), each bag $20 \mathrm{~g}, 2$ bags/time, 3 times/d; matched group30 cases of enteric-coated tablets, $0.5 \mathrm{~g} /$ time, 3 times/d. The course of treatment was 3 months. After 6 months of follow-up, the recurrence rate of the treatment group was8.00\%and that of the control group was $23.08 \%$. The recurrence rate between the two groups was significantly different $(\mathrm{P}<0.05)$. Qi Xiangfen [20] maintained relief by strengthening spleen and warming kidney acerbity intestines UC, 20 cases in the treatment group were given self-made Yu Kufang plus or minus oral (light attached tablets, Qing Dai, stir-fried Atractylodes macrocephala, Atractylodes macrocephala, cinnamon, elm, etc.), boiled water was taken $100 \mathrm{ml} /$ time, 2 times per day, and then gradually transitioned to the next day, every Tuesday dose. Control group 21 cases oral mesalazine sustained-release granules $500 \mathrm{mg} /$ time, 3 times per day. There were no adverse reactions during 1 year follow-up treatment, 15 cases in the treatment group and 10 cases in the control group. Wei Yongsheng [21] divided 78 patients into observation group 39 cases, give Jianpi Yuyang decoction (make fragrant, Poria cocos, stir-fried rice kernel, Codonopsis pilosula, etc.) water decoction 200 $\mathrm{ml}$, before meals; Control group 39 cases oral sulfasalazine ,1g/time, 4 times per day. Follow up for 3 months, the recurrence rate of the observation group was $25.6 \%$, the recurrence rate of the control group was $71.8 \%$, the difference between the two groups was statistically significant $(\mathrm{P}<0.05)$. Wang Chen [22] collected 50 patients who met the criteria of intake and discharge, and treated them with Jieyu Zhixie Fang (Bupleurum, White Peony, Angelica, Fried Atractylodes, Turmeric, etc.), 1 dose per day, $300 \mathrm{ml}$ each, early in the morning Take warm after dinner. After 4 weeks of treatment, 17 cases were effective, 28 cases were effective, 1 case was ineffective, and the total effective rate was $97.83 \%$, which was statistically significant compared with that before treatment $(\mathrm{P}<0.05)$. 


\subsection{Oral Chinese medicine combined with enema treatment}

Blood stasis and dampness remain in the intestinal mucosa injury, which is one of the factors UC the recurrence of remission. The retention enema of traditional Chinese medicine allows the medicine to be applied to the local area directly to the disease, fully exerting its efficacy, clearing the rat roots, making local inflammation subsided and lesion repair, and reducing oral administration to the stomach and possible liver damage.

Dong Wen [23] using the experience of regulating qi and blood stasis, clearing heat and removing dampness and detoxification as the method of oral enema combined with oral treatment of UC patients in the remission period, 20 cases of traditional Chinese medicine group were treated with Qinteng Yuyang decoction (baicalin, Chuanlian, Hongteng, Caulis chinensis, Baihe, etc.) every night before bedtime with enema and oral Changning decoction (Coptis chinensis, Scutellaria baicalensis, Zanthoxylum bungeanum, Codonopsis, Angelica sinensis, etc.) for 300 $\mathrm{ml}$ after meals. Matched group 20 cases oral mesalazine, $1.0 \mathrm{~g} /$ time, 2 times per day. After 3 courses of treatment, the TCM group was better than the control group in improving colonoscopy, mucosal pathological tissue and safety $(\mathrm{P}<0.05)$. Follow up for 3 months, the recurrence rate of the test group was 10 and the recurrence rate of the control group was 30. There was a significant difference between the two groups $(\mathrm{P}<0.05)$. Hu Chuanwen [24] to invigorate spleen and invigorate qi, Clearing heat and wet treatment of UC, in remission 40 oral mesalazine enteric-coated tablets in western medicine group, $0.5 \mathrm{~g} /$ time, 3 times per day; In Chinese medicine group, 40 cases were treated with Banxia Xiexin decoction, Codonopsis, Coptis, Ginger, Scutellaria baicalensis et al. Combined with traditional Chinese medicine retention enema (whiteweng, Sophora japonica, white and powder, Coptis chinensis, gallnut, etc.), Once a night. After the course of treatment, the total effective rate of traditional Chinese medicine group was $92.5 \%$, and that of western medicine group was $72.5 \%$, compared with the two groups $(\mathrm{P}<0.05)$; both groups can restore the balance of some intestinal flora, and the content of Escherichia coli in traditional Chinese medicine group was lower than that in western medicine group, and the content of Bifidobacterium and Lactobacillus was higher than that in western medicine group $(\mathrm{P}<0.05)$.

\subsection{Integrated Chinese and Western Medicine Treatment}

Modern Chinese medicine scientists combine the comprehensive judgment of UC patients' four diagnosis information with the characteristics of enteroscopy and pathology, and form the characteristics of macro and micro diagnosis and treatment of traditional Chinese and western medicine. Although western medicine has the disadvantages of high recurrence rate and many adverse reactions, its significant advantages in controlling disease progression cannot be ignored. Zhao Zhuangzhuang [25] a retrospective analysis showed that the remission period UC the addition of traditional Chinese medicine on the basis of western medicine treatment, can effectively reduce the recurrence rate of the disease and prolong the remission time. The combination of the two aims to make up for the deficiency and give full play to their respective therapeutic advantages in order to achieve the purpose of low toxicity and high efficiency.

Shang Lu [26] selected 92 patients with large intestinal damp-heat type UC as the research object. 48 patients in the treatment group were treated with Qinghua Lianyang Liangxue Decoction (Red Peony Root, Coptis, Pueraria lobata, Qin Pi, etc.) and Chinese medicine enema (Cork, Pulsatilla chinensis) during active period. Shichangpu, Sanyu, etc. treatment, while taking Edissa, $1 \mathrm{~g} /$ time, 4 times/d; Bacillus subtilis dual live bacteria enteric-coated capsules $500 \mathrm{mg} /$ time, 3 times/d; During the remission period, the patients were given Jianpi Qinghua Huoxue Prescription (Radix Astragali, Fried Atractylodes macrocephala, Fangfeng, Yunzhuan, etc.) and oral Aideisha, 2 g/d; The 44 cases in the control group received the above-mentioned western medicine treatment plan. Both groups were treated for 24 weeks. The follow-up to 32 weeks, the recurrence rates of treatment group and control group were $4.76 \%$ and $19.35 \%$, respectively. The difference was statistically significant $(\mathrm{P}<0.05)$, and the treatment group was better than the control group in improving the overall quality of life and social function $(\mathrm{P}<0.05)$. Zhou Minhong [27] to evaluate the efficacy of Jiawei Yupingfeng Powder in maintaining remission UC. 30 cases of control group were treated with mesalazine sustained-release granules g/ times and 2 times per day; on the basis of the control group, the treatment group was given Jiawei Yupingfeng Powder (Roasted Astragalus, Stir-fried Angelica, Stir-fried Atractylodes, Nutmeg, Stir-fried Fangfeng, etc.), 1 dose/day, orally 1 hour after breakfast and dinner, $200 \mathrm{ml}$ each time. After 48 weeks of treatment, the patients with no recurrence in the two groups were followed up for 15 to 65 months. The recurrence rate of the treatment group was $13.33 \%$, the recurrence rate of the control group was $43.33 \%$, and the two groups were compared by chi-square test $(\mathrm{P}<0.05)$. The long-term effect of Jiawei Yupingfeng Powder combined with western medicine was better than that of western medicine alone. 


\subsection{Other methods of treatment}

The characteristics and advantages of traditional Chinese medicine are not only reflected in individualization and safety, but also in its various treatment approaches and means. Acupuncture has a significant effect on the disease, and the treatment includes simple acupuncture, acupuncture and medicine. In addition, methods such as catgut embedding at acupoints, injection, and sticking have also made some progress in the treatment of this disease. Massage therapy regulates the autonomic nerve function of human body without increasing the extra pressure of spleen and stomach, reduces the secretion of intestinal mucosa, promotes the improvement of local microcirculation, and alleviates inflammatory reaction. No side effects and significant advantages.

Qu Manqing and other [28] with traditional Chinese medicine rectum drop combined with moxibustion treatment of damp-heat relief period UC, select points large intestine Yu and Feshu, each point moxibustion 20 min and with traditional Chinese medicine every night with drip method to retain enema (Baihe, Radix Paeoniae Alba, Angelica dahurica, Platycodon grandiflorum, Panax notoginseng powder, etc.), treatment for 2 weeks. Results of the 50 cases, 47 were cured, 3 were ineffective, and the total effective rate was 94\%. Liu Zhuhua et al. [29] selected patients with UC in remission, and 49 cases in the treatment group were treated with acupoint thread-embedding combined with traditional Chinese medicine enema. The acupoints were Tianshu, Dachangshu, Shenshu, Zusanli, etc. The intestinal line of sheep was buried under selected acupoints once in 20 days. Combined with traditional Chinese medicine enema (Pulsatilla, Sophora flavescens, Qingdai, Baihe, etc.) for 2 weeks, 46 cases in control group were treated with mesalazine granules for $2 \mathrm{~g} / \mathrm{d}$. After one year of follow-up, the total recurrence rate of the treatment group was 24.49 and that of the control group was 39.13\% $(\mathrm{p}<0.05)$. Yang Mengfan [30] the treatment of UC patients with damp-heat type and remission stage with massage combined with mesalazine. The results showed that the endoscopic mucosal and comprehensive curative effect was better than that of western medicine group $(\mathrm{P}<0.05)$.

\section{Summary}

Chinese medicine has the obvious advantage of improving individualized symptoms and regulating all-round functions. The combination of whole body and local "individualized comprehensive treatment" has brought into play the characteristics of holistic view of traditional Chinese medicine and individualized measures. It ensures the effectiveness and stability of long-term curative effect, improves clinical curative effect and reduces recurrence rate. While making good progress in the treatment of the disease, we still need to pay attention to its problems, such as small samples, non-blind, non-standard evaluation criteria, non-uniform follow-up time, drug use and dialectical classification, so the treatment of the disease still needs a long-term standard test, clinical demonstration to be objectively evaluated and improved. At present, these problems will be overcome with the unremitting efforts of Chinese medicine workers and the development of science and technology, and the prevention and treatment of UC remission period will be raised to a more satisfactory level.

\section{References}

[1] Cui Changwan, Sun Zhengrong. (2019). Advances in the pathogenesis of ulcerative colitis [J]; Modern Immunology, 2019, 39(01): 77-81.

[2] Yan Jianhua, Huang Guangrui. (n.d.). Advances in Modern Medical Research on the Causes and Pathogenesis of Ulcerative Colitis [J]. World Journal of Chinese Digestion, 27(04): 245-251.

[3] He Qiong, Li Jiandong. (2019). Advances in Epidemiology of Inflammatory Bowel Diseases [J]. Journal of Applied Medicine, 2019, 35(18): 2962-2966.

[4] Liu Dujia, Wang Yuanyuan, Ma Xu. (n.d.). Advances in Epidemiological Research on Ulcerative Colitis [J]. Chinese Journal of Burn Innovation, 29(03): 214-217.

[5] Zheng Kai, Shen Hong, Ye Bai. (n.d.). Discussion on the Diagnosis and Treatment of Ulcerative Colitis by Shenxi, Yangzi [J].

[6] Hou Yeting, Su Bingzhong, Chen Ping, et al. (n.d.). value of maintenance therapy in protracted period of ulcerative colitis [J]. Journal of Gastroenterology and Hepatology, 24(07): 855-857.

[7] Lu Guiling, Yan Ni, Cai Xiaohua. (2015). Importance of maintaining remission in reducing the recurrence rate of patients with ulcerative colitis [J]. Minkang Medicine, China, 2015, 27(21): 65-66, 77.

[8] Meng Xiaoyan, Cha Ansheng. (n.d.). On the mechanism and treatment progress of TCM recurrence in the remission stage of ulcerative colitis [J]. Clinical Journal of Traditional Chinese Medicine, 28(11): 1517-1519.

[9] Shu Qing, Yu Songren, Bai Yang, et al. (n.d.). Advances in immunological mechanism of spleen deficiency syndrome [J]. Journal of Jiangxi University of traditional Chinese Medicine, 30(06): 116-120. 
[10] Meng Fanzheng, Li Yannan, Zhao Jinsheng, et al. (n.d.). Advances in Modern Studies on the Essence of "Spleen Deficiency Syndrome” [J]. Shi Zhenguo Chinese Medicine, 30(12): 2975-2977.

[11] Huang Xiushen, Shen Tao, Liu Wei, Luo Yuxi, Wu Shiguo, Xu Wei, Hou Jianye, Su Xianhua, Zhang Ping. (2007). Effect of Pingwei Powder on Partial Immune Function of Spleen and Spleen Syndrome Model Rat [J]. Journal of Traditional Chinese Medicine, 2007(08): 730-732.

[12] Zhao Shengyun, Wang Dujie. (n.d.). The Effect of Sijunzi Decoction on T Cell Subgroup of Gastric Mucosa in Rats [J]. Spleen Deficiency Syndrome Chinese Journal of Integrated Chinese and Western Medicine Digestion, 22(04): 204-206.

[13] Yang Weiwen, Li Li, Tan Song, Liu Zhengyong, He Tianlan. (n.d.). Relationship between low-grade inflammatory response and recurrence of intestinal mucosa during the remission of ulcerative colitis [J]. Guizhou Medicine ,39(08): 734-736.

[14] Wang Wei. (2015). Characteristic dysbacteriosis [D]. In patients with inflammatory bowel disease during remission Wuhan University, 2015.

[15] Wen Xianghui, Liu Qiuping, Yu Yilan, et al. (n.d.). Relationship between internal dampness pathogenicity and intestinal flora dysbiosis [J]. Journal of Traditional Chinese Medicine, 59(16): 1377-1379.

[16] Wang Jing, Liu Xiaoyan, Huang Xiaoyu. (n.d.). Effects of external dampness on intestinal flora of rats [J]. Jilin traditional Chinese Medicine, 35(10): 1046-1049.

[17] Hu Yanyan, Shen Yonghua, Yu success, et al. (2017). Evaluation of blood hypercoagulability in patients with ulcerative colitis [J]. By coagulation function test Jiangsu Medicine, 2017, 43(11): 765-769.

[18] Han Lei. (2018). Analysis of Ulcerative Colitis and Thrombosis and Evaluation of Blood Hypercoagulability [D]. Methods Kunming Medical University.

[19] Zhang Tianbin, Qi Shuhao, Kuang Peng, et al. (n.d.). Clinical Observation of Jianpi Huoxue Jiedu Granule in Treatment of Relieving Ulcerative Colitis [J]. Relieving Period Chinese Journal of traditional Chinese Medicine, 34(11): 5491-5494.

[20] Qi Xiangfen. (n.d.). Clinical effect of self-made Yuku recipe on relieving ulcerative colitis with deficiency of spleen and kidney yang [D]. Liaoning University of traditional Chinese Medicine.

[21] Wei Yongsheng. (2017). Clinical study of Jianpi Yuxue Decoction in the treatment of ulcerative colitis in remission stage [J]. Asia Pacific Traditional Medicine, 2017, 13(14): 146-147.

[22] Wang Chen. (n.d.). Effect of Jieyu Zhixie Prescription on Relieving Chronic Ulcerative Colitis [D]. Observation Heilongjiang University of traditional Chinese Medicine.

[23] Dong Wen. (n.d.). Clinical study on the treatment of ulcerative colitis with Qindeng Yuyang Decoction based on "poison damage intestinal collaterals" [D]. Shandong University of traditional Chinese Medicine.

[24] Hu Chuanwen, Jiang Hongjuan, Zheng Xiaochun, et al. (2020). Effect of Banxia Xiexin decoction combined with retention enema on ulcerative colitis in remission stage and on intestinal flora level [J]. New Chinese Medicine, 2020, 52(03): 10-12.

[25] Zhao Zhuangzhuang. (2019). A Retrospective Analysis of 72 Cases of Ulcerative Colitis Treated with western medicine [D]. Anhui University of traditional Chinese Medicine.

[26] Shang Lu. (n.d.). Evaluation of the curative effect of integrated traditional Chinese and western medicine staging therapy for ulcerative colitis [D]. Nanjing University of traditional Chinese Medicine.

[27] Zhou Minhong. (n.d.). Evaluation of Effect of Jiawei Yupingfeng Powder on Relieving Ulcerative Colitis [D]. Nanjing University of traditional Chinese Medicine.

[28] Qu Manqing, Yin Ting, Wang Yipeng. (2018). Clinical observation on treatment of damp-heat ulcerative colitis with rectal drip combined with moxibustion [J]. Chinese Folk Therapy, 2018, 26(08): 39-40.

[29] Liu Zhuhua, Ren Shunping, Hao Hairong, et al. (n.d.). Effect of acupoint catgut embedding combined with traditional Chinese medicine enema on relieving ulcerative colitis [J]. Observation Journal of Shanxi Institute of traditional Chinese Medicine 14(04):43-45.

[30] Yang Mengfan. (n.d.). Effect of massage combined with western medicine on protracted period of damp-heat ulcerative colitis [D]. Nanjing University of traditional Chinese Medicine. 